\title{
Integrated weighted gene coexpression network analysis identifies Frizzled 2 (FZD2) as a key gene in invasive malignant pleomorphic adenoma
}

\author{
Zhenyuan Han ${ }^{1,2,3 \dagger}$, Huiping Ren ${ }^{4 \dagger}$, Jingjing Sun ${ }^{1,2+}$, Lihui Jin ${ }^{5}$, Qin Wang ${ }^{6 *}$, Chuanbin Guo ${ }^{3^{*}}$ and Zhen Tian ${ }^{1,2^{*}}$ (1)
}

\begin{abstract}
Background: Invasive malignant pleomorphic adenoma (IMPA) is a highly malignant neoplasm of the oral salivary glands with a poor prognosis and a considerable risk of recurrence. Many disease-causing genes of IMPA have been identified in recent decades (e.g., P53, PCNA and HMGA2), but many of these genes remain to be explored. Weighted gene coexpression network analysis (WGCNA) is a newly emerged algorithm that can cluster genes and form modules based on similar gene expression patterns. This study constructed a gene coexpression network of IMPA via WGCNA and then carried out multifaceted analysis to identify novel disease-causing genes.
\end{abstract}

Methods: RNA sequencing (RNA-seq) was performed for 10 pairs of IMPA and normal tissues to acquire the gene expression profiles. Differentially expressed genes (DEGs) were screened out with the cutoff criteria of $\| \log _{2}$ Fold change $(F C) \mid>1$ and adjusted $p$ value $<0.05$. Then, WGCNA was applied to systematically identify the hidden diagnostic hub genes of IMPA.

Results: In this research, a total of 1970 DEGs were screened out in IMPA tissues, including 1056 upregulated DEGs and 914 downregulated DEGs. Functional enrichment analysis was performed for identified DEGs and revealed an enrichment of tumor-associated GO terms and KEGG pathways. We used WGCNA to identify gene module most relevant with the histological grade of IMPA. The gene FZD2 was then recognized as the hub gene of the selected module with the highest module membership (MM) value and intramodule connectivity in protein-protein interaction (PPI) network. According to immunohistochemistry (IHC) staining, the expression level of FZD2 was higher in low-grade IMPA than in high-grade IMPA.

\footnotetext{
*Correspondence: sylarair601@163.com; guodazuo@sina.com; tianzhen_256@126.com

${ }^{\dagger}$ Zhenyuan Han, Huiping Ren and Jingjing Sun are co-first authors and contributed equally to this work.

${ }^{1}$ Department of Oral Pathology, Shanghai Ninth People's Hospital,

Shanghai Jiao Tong University School of Medicine, Shanghai, China

${ }^{3}$ Department of Oral and Maxillofacial Surgery, Peking University School

and Hospital of Stomatology, Beijing, China

${ }^{6}$ Clinical Translational Research Center, Shanghai Pulmonary

Hospital, School of Life Sciences and Technology, Tongji University,

Shanghai 200092, China

Full list of author information is available at the end of the article
}

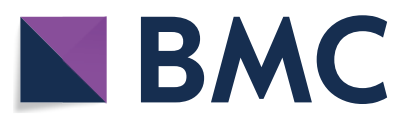

(c) The Author(s) 2022. Open Access This article is licensed under a Creative Commons Attribution 4.0 International License, which permits use, sharing, adaptation, distribution and reproduction in any medium or format, as long as you give appropriate credit to the original author(s) and the source, provide a link to the Creative Commons licence, and indicate if changes were made. The images or other third party material in this article are included in the article's Creative Commons licence, unless indicated otherwise in a credit line to the material. If material is not included in the article's Creative Commons licence and your intended use is not permitted by statutory regulation or exceeds the permitted use, you will need to obtain permission directly from the copyright holder. To view a copy of this licence, visit http://creativecommons.org/licenses/by/4.0/. The Creative Commons Public Domain Dedication waiver (http://creativeco mmons.org/publicdomain/zero/1.0/) applies to the data made available in this article, unless otherwise stated in a credit line to the data. 
Conclusion: FZD2 shows an expression dynamic that is negatively correlated with the clinical malignancy of IMPA and it plays a central role in the transcription network of IMPA. Thus, FZD2 serves as a promising histological indicator for the precise prediction of IMPA histological stages.

Keywords: Invasive malignant pleomorphic adenoma (IMPA), Weighted gene coexpression network analysis (WGCNA), FZD2

\section{Background}

Invasive malignant pleomorphic adenoma (IMPA) is characterized by high malignancy and invasive growth. It is a subtype of carcinoma ex pleomorphic adenoma (Ca-ex-PA) that arises mainly in the parotid gland, with more than $1.5 \mathrm{~mm}$ of cancerous components extending beyond the adenoma capsule into surrounding tissues $[1,2]$. Based on the histological morphology of the malignant components, IMPA can be subclassified into the myoepithelial subtype and adenocarcinoma subtype. Briefly, if two or more tumor myoepithelial markers, such as Calponin, S100, or SMA are positively expressed at the same time, IMPA is considered to be a myoepithelial carcinoma subtype. If tumors do not meet the above criteria or are only positive for the epithelial marker Ckpan, they are categorized as adenocarcinoma subtypes [3, 4]. To date, surgical resection combined with radiotherapy and chemotherapy is the main treatment strategy, but the prognosis is poor due to higher local recurrence, distant metastasis and a lower survival rate after surgery $[1,5,6]$. Thus, further research on more accurate molecular targets for early diagnosis and targeted therapy and predictors of a good prognosis of IMPA are urgently needed to develop more efficient therapy that can improve patient survival and quality of life.

A diffusion-weighted imaging (DWI)-based triple-classification radiomics model to characterize intratumoral heterogeneity for preoperative auxiliary diagnosis of pleomorphic adenoma (PA) and a nomogram for predicting the prognosis in an individual with Ca-ex-PA have been established [7]. However, these models are limited to neoplasms and more precise early diagnostic biomarkers are necessary [8]. As bioinformatics analysis is widely applied to cancer research, our previous study first profiled the $\mathrm{N}^{6}$-methyladenosine $\left(\mathrm{m}^{6} \mathrm{~A}\right)$ methylome map in IMPA by methylated RNA immunoprecipitation with high-throughput sequencing (MeRIP-seq) [9]. This study indicated a significant effect of $\mathrm{m}^{6} \mathrm{~A}$ modification on IMPA progression, but gene-targeted diagnostic biomarkers are not clear. Weighted gene coexpression network analysis (WGCNA) is a suitable tool to establish free-scale coexpression networks and it is utilized to determine the hub genes based on the correlation between gene modules and clinical characteristics [10, 11]. It has been successfully used in a variety of tumor studies. With WGCNA, potential biomarkers and molecular mechanisms of breast cancer, papillary thyroid carcinoma (PTC) and medulloblastoma (MB) etc. have been identified [12-14], but a gene coexpression network for identifying hub genes closely related to IMPA is still poorly characterized.

Therefore, we evaluated the relationship between the hub gene and tumor grade using WGCNA since histological grade has a strong effect on diagnosis, treatment and prognosis. To the best of our knowledge, our research is the first to utilize WGCNA for the underlying mechanism profiling and biomarker verification of IMPA, which might provide new ideas for increasing diagnosis accuracy and designing efficient strategies for IMPA.

\section{Methods}

\section{Tissue samples}

Ten pairs of IMPA and adjacent normal control tissues for RNA sequencing (RNA-seq) were obtained from Shanghai Ninth People's Hospital. In addition, another 45 Formalin-fixed and paraffin-embedded IMPA tissues with clinicopathological information were retrieved for verification of hub genes via Immunohistochemistry (IHC). The clinicopathological information of these 45 tissue samples is summarized in Table 1. This study was approved by the ethics committee of Shanghai Ninth People's Hospital.

\section{Histological analyses}

All IMPA tissues and adjacent normal controls were fixed in $4 \%$ PFA for $>4 \mathrm{~h}$ at $4{ }^{\circ} \mathrm{C}$. Then, these fixed tissues were dehydrated with graded ethanol (70-100\%) and embedded in paraffin. Sections $(4 \mu \mathrm{m})$ were cut on a Leica HistoCore BIOCUT RM2235. Hematoxylin-eosin (H\&E) staining was performed following standard procedures. Stained sections were imaged using a Leica LF200 microscope.

\section{IHC staining}

IHC staining was carried out as previously described [15]. In brief, tissue Sections $(4 \mu \mathrm{m})$ were dewaxed in xylene twice for 2 min each and then rehydrated in a graded series of ethanol (100-70\%). Antigen retrieval was performed by boiling sections for $15 \mathrm{~min}$ in sodium citrate buffer $(10 \mathrm{mM}$ citrate acid, $10 \mathrm{mM}$ sodium citrate, 
Table 1 Clinicopathological characteristics of IMPA samples used for FZD2 IHC staining

\begin{tabular}{lll}
\hline Characteristics & Category & Number of cases (\%) \\
\hline Age (years) & $<60$ & $14(31.1 \%)$ \\
Gender & $\geq 60$ & $31(68.9 \%)$ \\
Site & Female & $10(22.2 \%)$ \\
& Male & $35(77.8 \%)$ \\
Subtype & Major salivary gland & $40(88.9 \%)$ \\
& Minor salivary gland & $5(11.1 \%)$ \\
& Adenocarcinoma & $21(46.7 \%)$ \\
Grade & subtype & \\
& Myoepithelial subtype & $24(53.3 \%)$ \\
Lymph node metastasis & Yes & $29(64.4 \%)$ \\
& High & $16(35.6 \%)$ \\
Perineural invasion & Yes & $18(40.0 \%)$ \\
& No & $27(60.0 \%)$ \\
Expression of FZD2 & Low expression & $28(62.2 \%)$ \\
& High expression & $21(53.3 \%)$ \\
\hline
\end{tabular}

$\mathrm{pH}$ 6.0). Then, $5 \%$ normal donkey serum was used to block nonspecific antigens. IHC was performed using the Dako EnvisionTM method for antibody incubation and then developed by using the DAB peroxidase substrate kit (Beyotime, P0202). IHC-stained sections were imaged by a Leica LF200 microscope.

Antibodies for IHC included anti-S100 (Dako Z0311, 1:200), anti-Calponin (Dako M3556, 1:100), anti-SMA (Dako M0851, 1:100), anti-CK7 (Dako M7018, 1:50) and anti-CK19 (Dako M0888, 1:100), anti-FZD2 (Bioworld BS3163, 1:50), which were purchased from commercial sources.

\section{RNA-seq and differentially expressed gene (DEG) identification}

Ten pairs of IMPA tissue samples and the surrounding normal control were immediately placed into RNase-free centrifuge tubes and snap-frozen in liquid nitrogen for RNA-seq. Briefly, total RNA was extracted and controlled for quality using a BioAnalyzer 2100 system (Agilent Technologies, Inc., USA). Then, RNA-seq was performed by Illumina NovaSeq ${ }^{\mathrm{TM}} 6000$. EdgeR was performed to normalize the raw data [16] and the DEGs were identified via adjusted $\mathrm{p}$ value and Fold change (FC).

\section{Functional and pathway enrichment analysis}

The acquired DEGs were analyzed by the database for annotation, visualization and integrated discovery (DAVID) database (https://david.ncifcrf.gov/) [17, 18] online tool for gene ontology (GO) [19] enrichment analysis and Kyoto Encyclopedia of Genes and Genomes (KEGG) [20] pathway enrichment analysis to identify potential biological functions of DEGs in IMPA.

\section{Weighted gene co-expression network construction}

The R package 'WGCNA' was used to construct a gene coexpression network of DEGs [10]. The correlation strength between nodes was calculated using an adjacency matrix and the formula was as follows:

$$
\mathrm{s} i j=|\operatorname{cor}(\mathrm{x} i, \mathrm{x} j)| \mathrm{a} i j=\mathrm{S} i j \beta
$$

Briefly, $i$ and $j$ in the formula were two distinct genes and $x i$ and $x j$ represented their expression values. sij is Pearson's correlation coefficient and aij is the strength of the correlation between two genes. The soft-threshold $\beta$ was 10 in this study. Then, we transformed the adjacency matrix into the topological overlap matrix and performed hierarchical clustering to identify modules with min. Module Size $=30$. Notably, genes without characteristics were assigned to the grey module. Subsequently, gene significance (GS), module membership (MM), module eigengene (ME) and other parameters were calculated to identify the module most relevant with histological grade of IMPA.

\section{Identification and validation of hub genes}

Genes in the selected clinically relevant module were uploaded to the Search Tool for the Retrieval of Interacting Genes/Proteins (STRING) (https://string-db.org/) $[21,22]$ to analyze the protein-protein interaction (PPI) network of DEGs. The PPI network was visualized via Cytoscape software [23]. KEGG pathway enrichment of these genes was analyzed with the R package 'ClusterProfiler' to investigate the biological functions of the module [24]. Of note, the gene with $M M>0.8$ and the highest degree of connectivity was determined to be the hub gene. IHC was further performed to examine the expression level of the hub gene in different clinical stages of IMPA.

\section{Statistical analysis}

Data were expressed as mean \pm standard deviation (SD) and analyzed using SPSS version 23 statistical analysis package (SPSS Inc., Chicago, IL, USA). Unpaired/paired Student's t test (two-tailed) was applied to analyze differences between two groups. Pearson's chi-square test was used to assess differences between the low-grade and high-grade groups. The Pearson's correlation coefficient analysis was performed to examine the correlation. Statistical significance was described as follows: "p value $<0.05$; **"p value $<0.01$; *** p value $<0.001$. 


\section{Results}

\section{Pathologic subtypes of IMPA}

IMPA can be divided into the myoepithelial subtype and the adenocarcinoma subtype [4]. In the 45 IMPA samples for IHC staining, there were 24 myoepithelial IMPAs, which were $\mathrm{S} 100^{+} \mathrm{SMA}^{+}$Calponin ${ }^{+} \mathrm{CK} 7^{-} \mathrm{CK} 19^{-}$ (Fig. 1) and 21 adenocarcinoma IMPAs, which were $\mathrm{CK}^{+}{ }^{+} \mathrm{CK} 19^{+} \mathrm{S}_{100}{ }^{-} \mathrm{SMA}^{-} \mathrm{Calponin}^{-}$(Fig. 2).

\section{Identification of DEGs}

Based on $\left|\log _{2} \mathrm{FC}\right|>1$ and adjusted $\mathrm{p}$ value $<0.05$, a total of 1970 DEGs between IMPA and normal samples were screened out, including 1056 upregulated and 914 downregulated genes (Fig. 3a) [25]. In addition, volcano plots listed the top 10 upregulated and downregulated genes (Fig. 3b, Table 2). More details can be found in Additional file 1: Table S1.

\section{Functional and pathway enrichment analysis of DEGs}

GO and KEGG pathway enrichment analyses were used to verify the potential functional and molecular pathways associated with DEGs. The upregulated DEGs were significantly enriched in biological process of GO terms including nuclear division, organelle fission, extracellular matrix (ECM) organization and chromosome segregation (Fig. 4a). On the other hand, downregulated DEGs were mainly involved in second messenger-mediated signaling, lipid catabolic processes and purine-containing compound catabolic processes (Fig. 4b). In addition, KEGG pathway analysis revealed that upregulated DEGs exhibited enrichments in the cell cycle, ECM-receptor interaction and p53 signaling pathway (Fig. 4c), whereas downregulated DEGs were mainly enriched in salivary secretion, calcium signaling and cAMP signaling pathways (Fig. 4d).

In general, GO and KEGG pathway analyses both suggestd that upregulated DEGs in IMPA exerted functions of promoting tumorigenesis. They were involved in cell cycle progression, ECM organization and tumor-associated signaling regulation. Similarly, downregulated DEGs were revealed to participate in the modulation of second messenger-mediated signaling, which is extremely important for oncogenesis. Notably, the KEGG pathway analysis of downregulated DEGs also showed enrichments in second messenger-mediated signaling (e.g., cAMP and calcium), indicating a critical role of second messengers in IMPA pathogenesis.

\section{Identification of gene module relevant with IMPA histological grade}

A cluster dendrogram of 1970 DEGs was produced via WGCNA based on the criteria of soft-threshold $\beta=10$ and scale-free $\mathrm{R}^{2}=0.85$ (Fig. $5 \mathrm{a}-\mathrm{c}$ ). Then, 11 gene modules were identified in the hierarchical clustering, based on a merge cut height of 0.25 and a minimum module size of 30 (Figs. 5d, 6a). The enriched GO terms and KEGG pathways of these 11 gene modules are summarized in Additional file 2: Table S2. It was intriguing to find that there were 6 modules exhibiting enrichment of tumor-associated signaling pathways or GO terms, namely, the cyan module, green module, grey60 module, midnight blue module, salmon module and yellow module. Reasonably, those modules were significantly relevant to the onset and progression of IMPA. The remaining 5 gene modules did not show tumor-related pathway or GO enrichments and thus were not deeply explored in this study.

Among the 6 tumor-associated modules, the green one showed the strongest correlation with the histological grade of IMPA ( $R=-0.62 ; \mathrm{p}=0.06$; Fig. 6b). Besides, the green module had the highest correlation with histological grade in GS analysis (Fig. 6c). It was noteworthy that MM and GS of the green module were positively related (Fig. 6d). Therefore, the green module was selected for further analysis.

\section{FZD2 as a promising biomarker of IMPA malignancy}

KEGG pathway analysis was performed to determine the possible functions of the 132 genes in the green module. As a result, the 132 genes in the green module were primarily enriched in pathways of the Wnt signaling pathway, protein digestion and absorption and ECM-receptor interaction (Fig. 7a). Subsequently, the PPI network of the green module was constructed via the STRING database (Fig. 7b). Notably, FZD2 had the highest MM value and intramodule connectivity among genes involved in the PPI network (Table 3; Additional file 3: S3) and was therefore selected as the core gene. IHC staining of FZD2 revealed that the expression level of FZD2 was negatively correlated with the clinical malignancy of IMPA; that is, the FZD2 protein level was lower in high-grade IMPA than in low-grade IMPA. FZD2 IHC staining was then performed in distinct grades of IMPA tissues and showed identical results (Fig. 7c-e; Table 4). These results suggest that FZD2 might be an ideal indicator for predicting the histological stages of IMPA.

\section{Discussion}

IMPA is a seriously malignant salivary gland neoplasm with a high risk of recurrence and poor prognosis [1, 2], especially in high-grade tumors [8]. Thus, better biomarkers are needed and the underlying pathogenesis must be clarified for precise diagnosis, targeted treatment and the prognosis prediction of IMPA. Therefore, 10 pairs of IMPA tissue and tumor-surrounding normal 

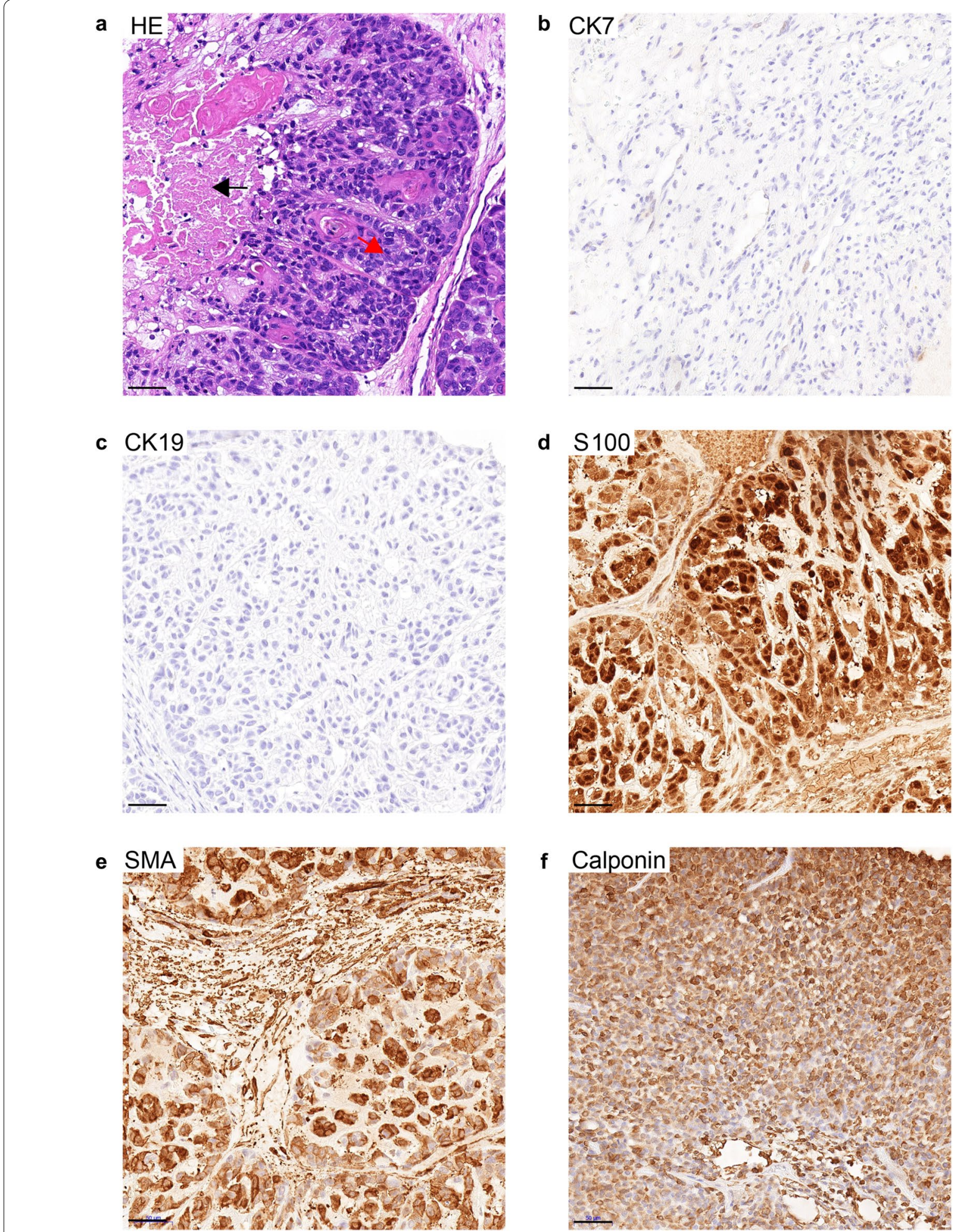

Fig. $1 \mathrm{H} \& \mathrm{E}$ and IHC staining in the myoepithelial subtype of IMPA. a H\&E staining. Tumor cells were arranged in nodule patterns (red arrow) and separated by thick fibrous stroma. Necrosis (black arrow) was observed in the center of the tumor. IHC staining of CK7 (b), CK19 (c), S100 (d), SMA (e) and Calponin (f). The myoepithelial subtype of IMPA was positive for S100, SMA and Calponin, but was negative for CK7 and CK19. Scale bar $=50 \mu \mathrm{m}$ 
a

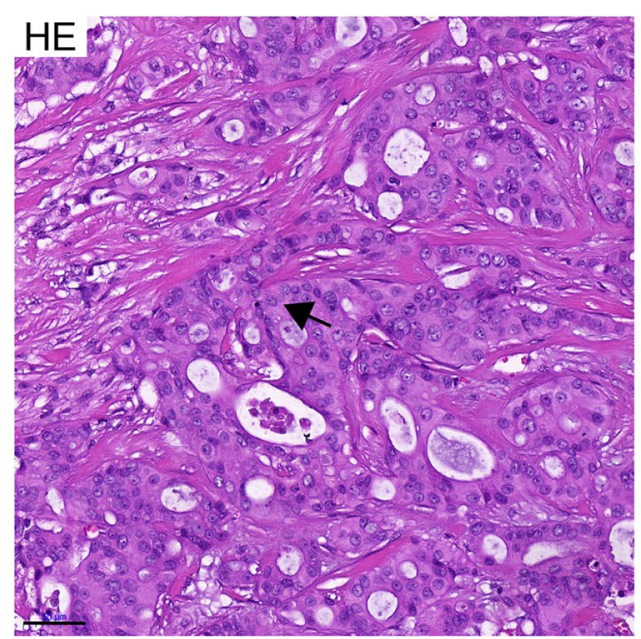

C

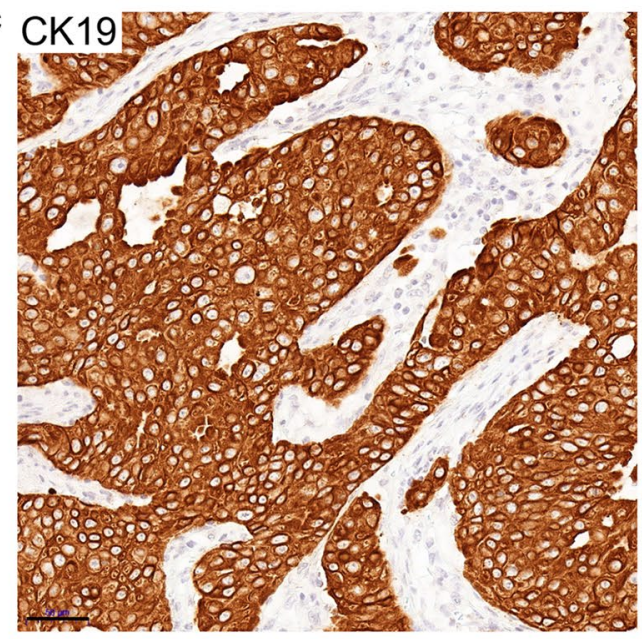

e SMA

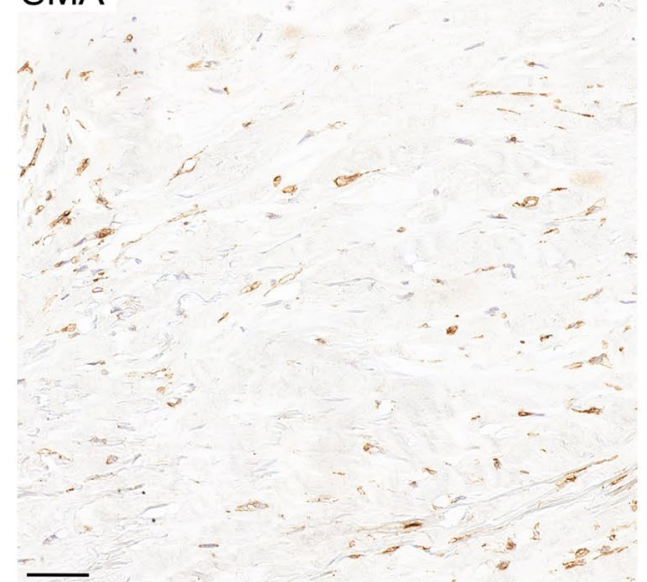

b

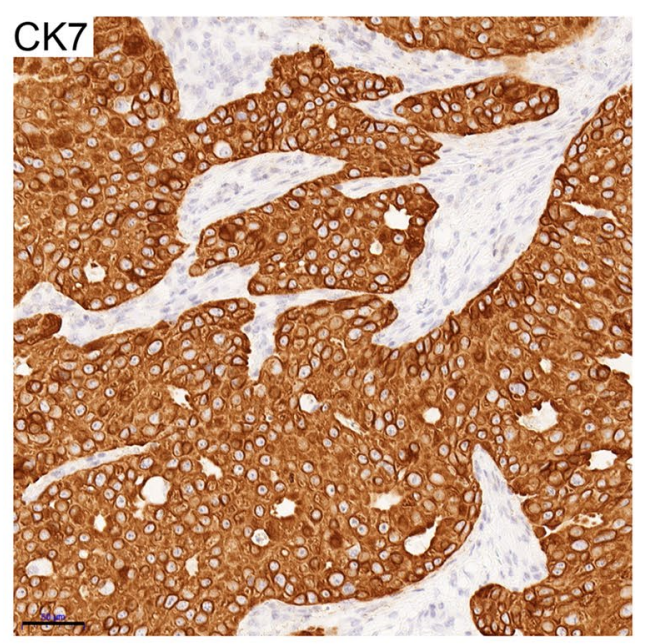

d $\mathbf{S 1 0 0}$

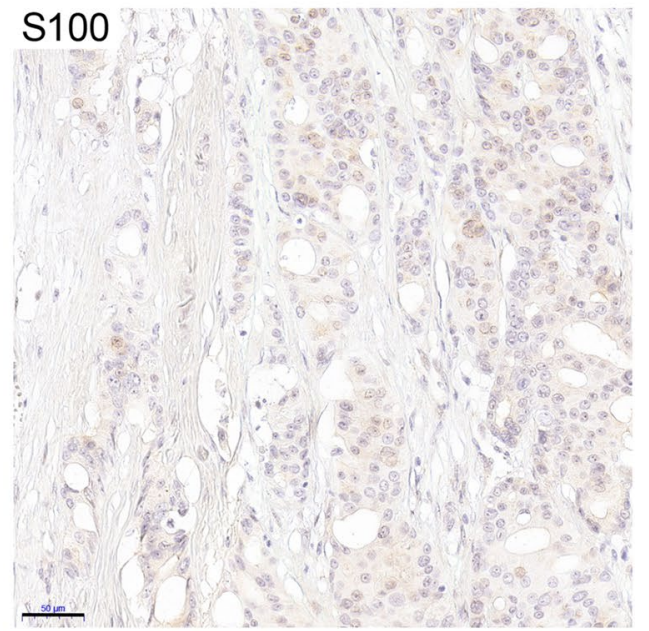

f Calponin

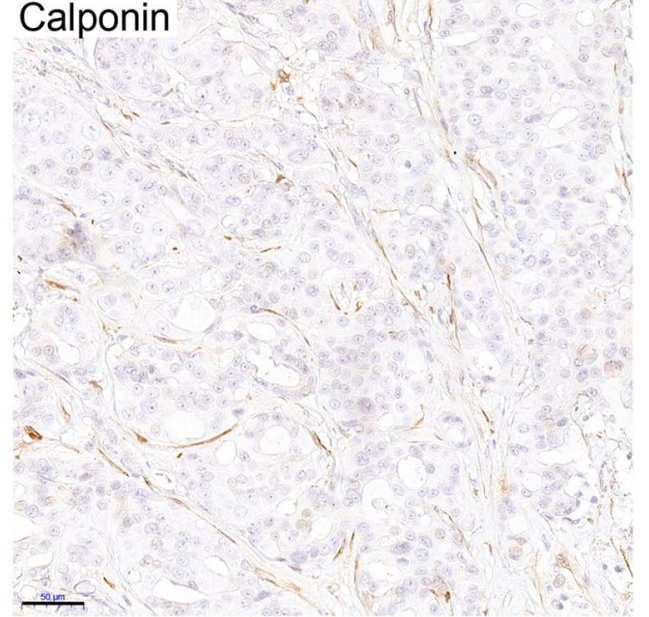

Fig. 2 H\&E and IHC staining in the adenocarcinoma subtype of IMPA. a H\&E staining. Tumor cells were arranged in ductal and nested structures (black arrow), with abundant eosinophilic cytoplasm and prominent atypia of cells and nuclei. IHC staining of CK7 (b), CK19 (c), S100 (d), SMA (e) and Calponin (f). The adenocarcinoma subtype of IMPA was positive for CK7 and CK19, but was negative for S100, SMA and Calponin. Scale bar $=50 \mu \mathrm{m}$ 

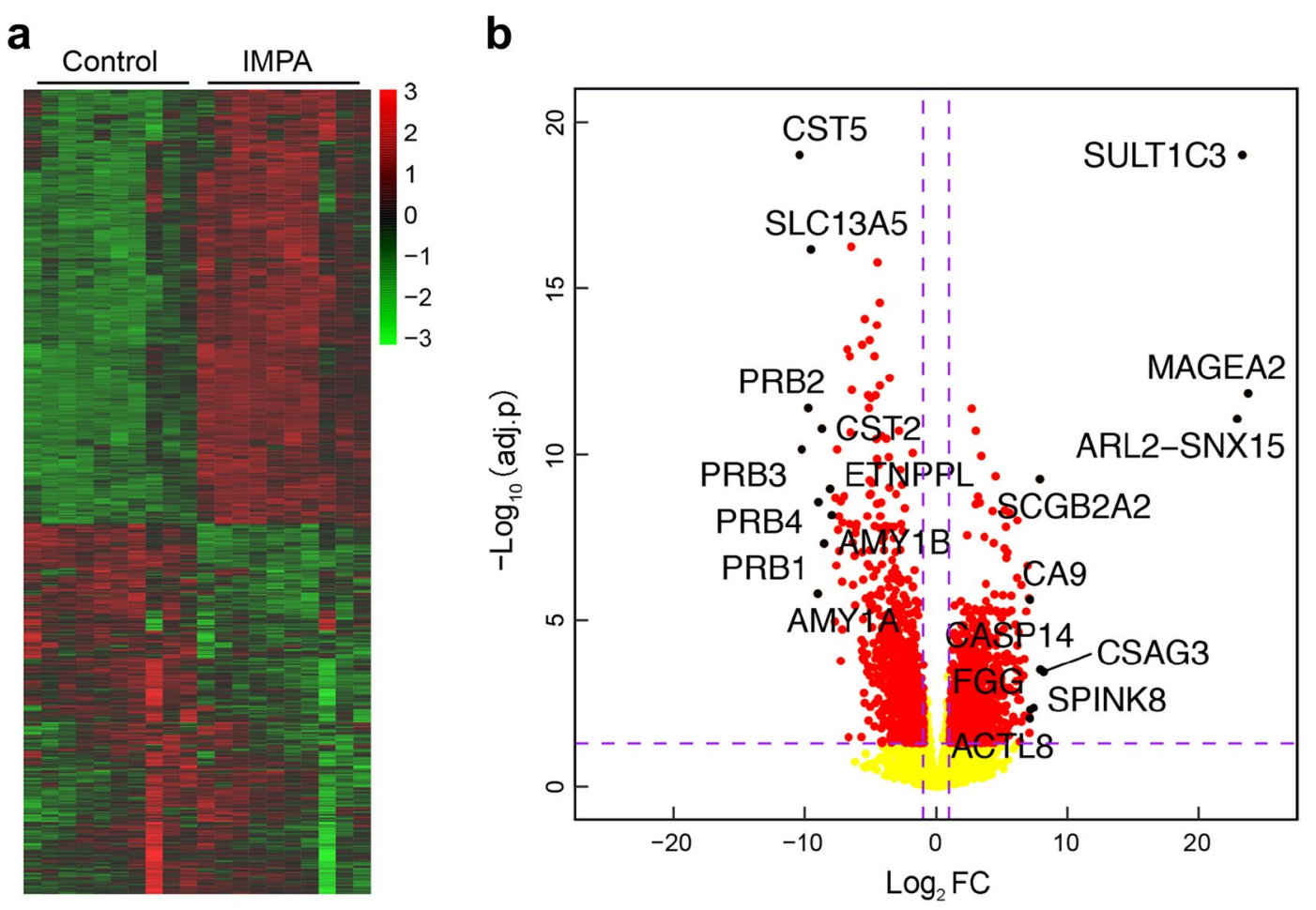

Fig. 3 Heatmap and volcano plot displaying the 1970 DEGs in IMPA and adjacent normal controls. a The heatmap plot (red, upregulated; green, downregulated). The original data were normalized via the $z$-score to indexes between -3 and 3. b The volcano plot (adjusted $p$ value $<0.05$, $\left|\log _{2} \mathrm{FC}\right|>1$; red squares). The $\mathrm{p}$ value was adjusted using the Benjamini-Hochberg procedure

Table 2 The top 10 upregulated DEGs and downregulated DEGs

\begin{tabular}{lcl}
\hline Gene_symbol & Log $_{2}$ FC & adjusted $\mathbf{p}$ value \\
\hline MAGEA2 & 23.71767826 & $1.48 \mathrm{E}-12$ \\
SULT1C3 & 23.27972921 & $9.83 \mathrm{E}-20$ \\
ARL2-SNX15 & 22.89375721 & $8.54 \mathrm{E}-12$ \\
CSAG3 & 8.151055524 & 0.000359128 \\
CASP14 & 7.905691636 & 0.000307196 \\
SCGB2A2 & 7.86520853 & $5.69 \mathrm{E}-10$ \\
SPINK8 & 7.381732165 & 0.004260212 \\
FGG & 7.100609633 & 0.004865952 \\
CA9 & 7.072063302 & $2.36 \mathrm{E}-06$ \\
ACTL8 & 7.062711902 & 0.009017509 \\
AMY1B & -8.017880777 & $6.76 \mathrm{E}-09$ \\
ETNPPL & -8.146282116 & $1.11 \mathrm{E}-09$ \\
PRB1 & -8.596065483 & $4.87 \mathrm{E}-08$ \\
CST2 & -8.759797112 & $1.73 \mathrm{E}-11$ \\
PRB4 & -9.022705183 & $2.75 \mathrm{E}-09$ \\
AMY1A & -9.068037572 & $1.57 \mathrm{E}-06$ \\
SLC13A5 & -9.586123392 & $6.87 \mathrm{E}-17$ \\
PRB2 & -9.787907831 & $4.01 \mathrm{E}-12$ \\
PRB3 & -10.29818453 & $7.14 \mathrm{E}-11$ \\
CST5 & -10.46657151 & $9.83 \mathrm{E}-20$ \\
\hline
\end{tabular}

tissue samples were subjected to RNA-seq analysis in this study.

In our study, 1970 DEGs were identified according to the criteria of $\left|\log _{2} \mathrm{FC}\right|>1$ and adjusted $\mathrm{p}$ value $<0.05$. These DEGs included 1056 upregulated genes and 914 downregulated genes. According to the GO analysis results, upregulated DEGs were mainly enriched in nuclear division and extracellular matrix (ECM) organization. The upregulated DEGs, as revealed by KEGG enrichment analysis, participated in pathways such as ECM-receptor interaction and p53 signaling. The ECM provides biochemical and essential structural support for cellular constituents of the tissue and is responsible for cell proliferation, cell adhesion and cell-cell communication [25-27]. Aberrant ECM organization has been reported to be associated with the progression of multiple tumors (e.g., breast cancer and hepatocellular carcinoma) and may likewise contribute to IMPA development [28, 29]. Furthermore, the downregulated DEGs exhibited an enrichment in the GO category of second messenger-mediated signaling and were involved in salivary secretion and calcium signaling pathways. Notably, intracellular calcium ions $\left(\mathrm{Ca}^{2+}\right)$ are well-studied second messengers and they play direct and robust roles in many biological processes. Calcium 

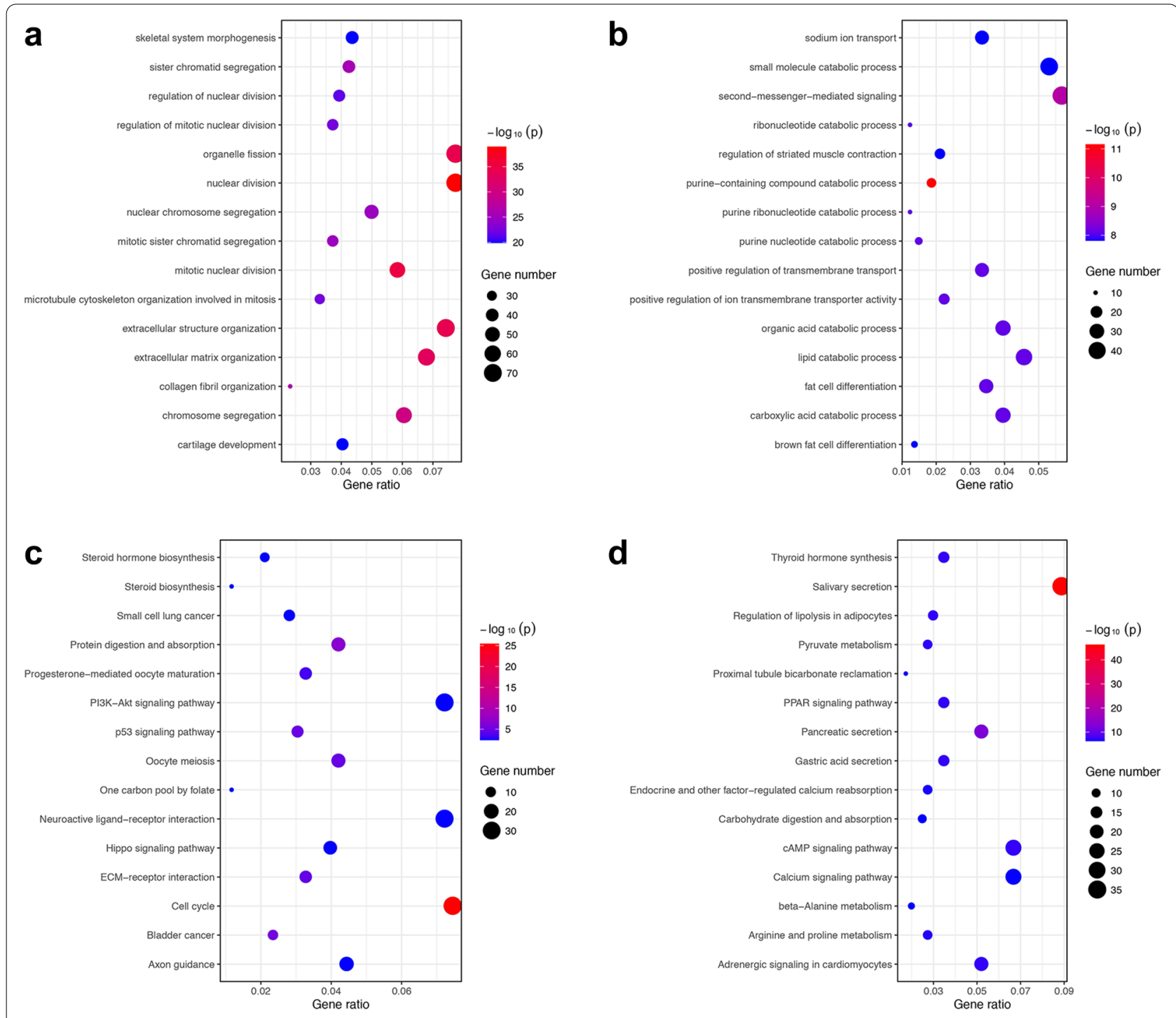

Fig. 4 The top 15 ranked GO terms and KEGG pathways according to ' $-\log _{10}(p$ value)'. Bubble plots displaying the top 15 enriched GO terms of biological process in 1056 upregulated DEGs (a) and 914 downregulated DEGs (b). Bubble plots displaying the top 15 enriched KEGG pathways in 1056 upregulated DEGs (c) and 914 downregulated DEGs (d)

signaling changes reflect the 'in or out' of intracellular $\mathrm{Ca}^{2+}[30,31]$. Studies have revealed that calcium signaling regulates cancer progression mainly by modulating immune-associated pathways and remodeling the tumor microenvironment [32,33]. Therefore, there can be dysregulation of intracellular $\mathrm{Ca}^{2+}$ and calcium signaling in IMPA.

To determine the hub genes of the 1970 DEGs, WGCNA was performed to construct a gene coexpression network associated with the clinical features of IMPA. Among the 11 identified gene modules, the green module, comprised of 132 DEGs, had the strongest correlation with IMPA stage and grade and was selected for further analysis. Enrichment analysis showed that genes in the green module were enriched in the Wnt signaling pathway, protein digestion and absorption, ECM-receptor interaction and neuroactive ligand receptor interaction. The Wnt signaling pathway is a critical pathway in multiple biological processes [34]. Aberrant activity of the Wnt pathway plays an important role in carcinogenesis, cancer proliferation, metastasis and invasion [35]. Moreover, the activation of protein anabolism is important to high-grade cancers because proteins are required for cell division during 


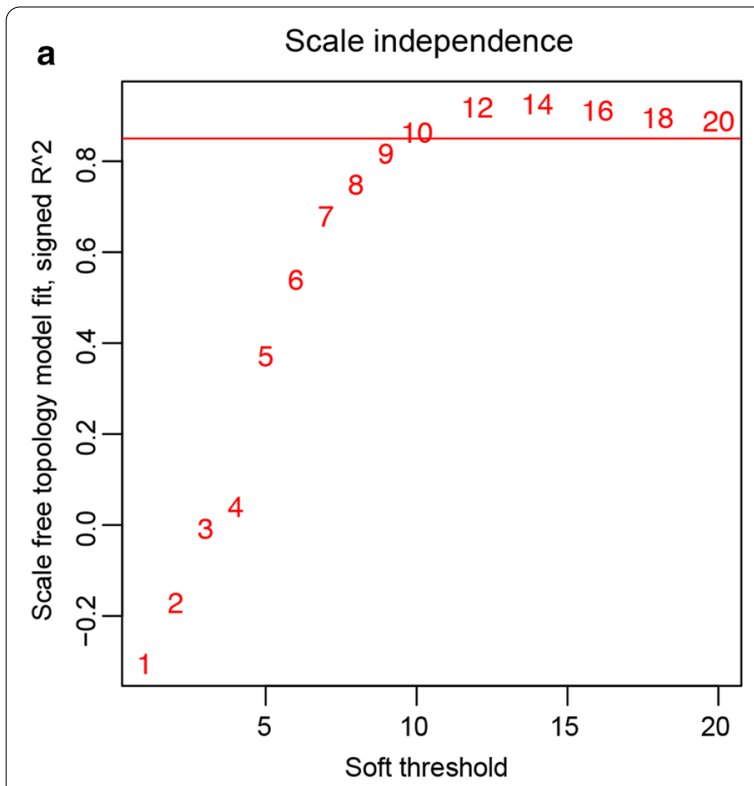

C Histogram of $\mathrm{k}$

Check scale free topology

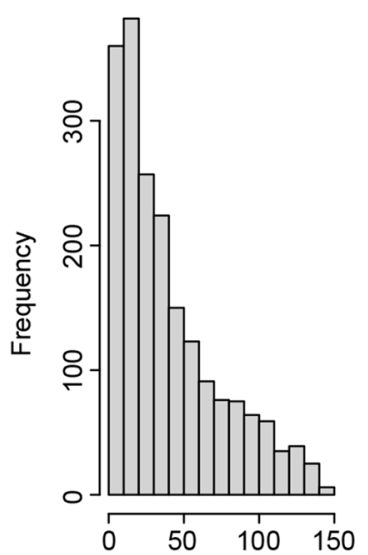

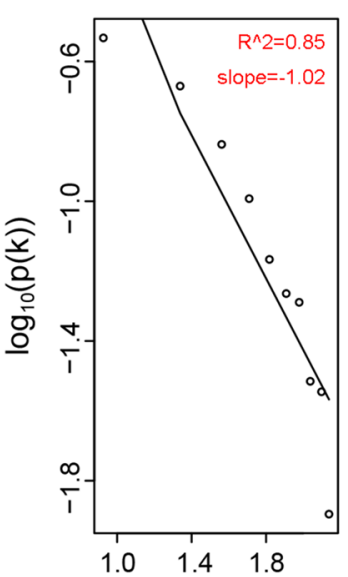

b

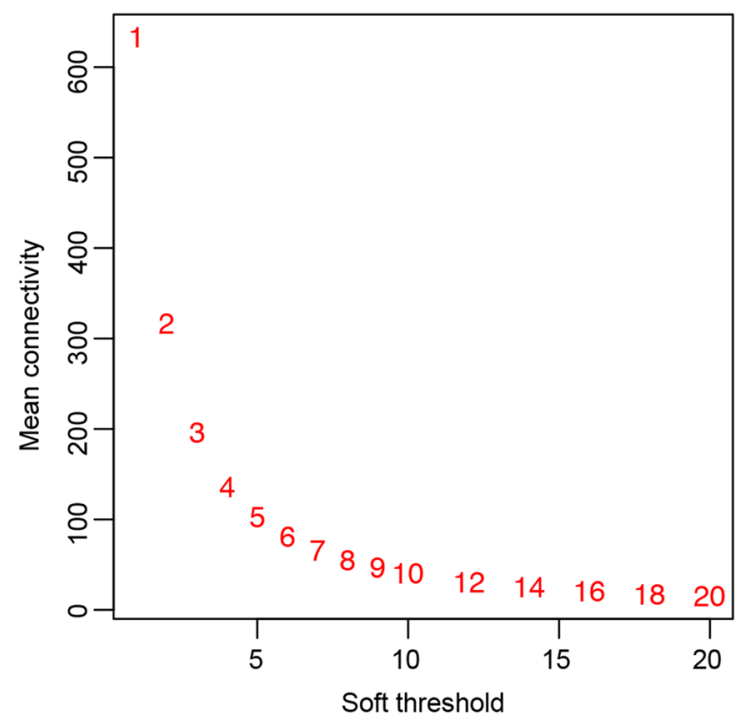

d

Cluster Dendrogram

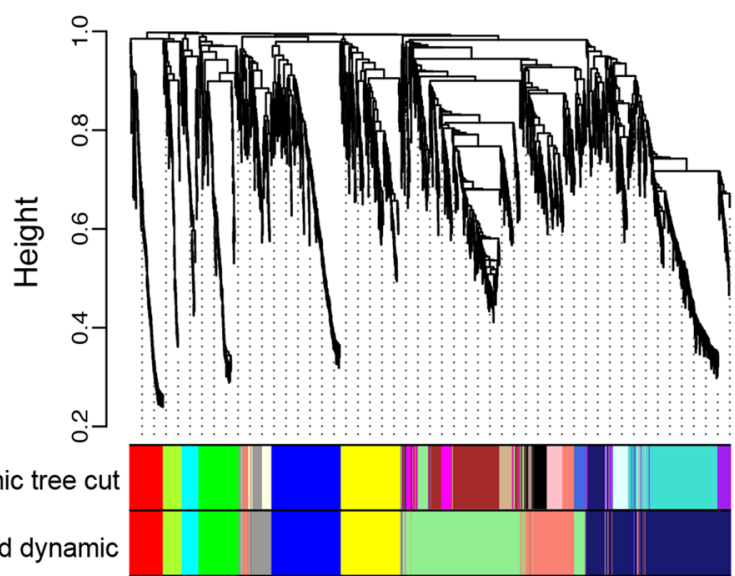

Fig. 5 WGCNA network and module detection. a Scale-free fit index (y-axis) as a function of soft-thresholding power ( $x$-axis). Power 10 was chosen because the fit index curve flattened out upon reaching a high value $(>0.85)$. $\mathbf{b}$ The mean connectivity $(y$-axis) as a function of soft-thresholding powers (x-axis). c Checking the scale-free topology for the soft-thresholding power chosen. $\mathbf{d}$ Module assignment and cluster dendrogram. Highly interconnected groups of genes were clustered, and modules were represented by distinct colors in the horizontal bar. Eleven modules were identified with the hierarchical clustering tree analysis

proliferation and against cancer-associated cachexia and malnutrition [36]. Studies have reported that neuroactive ligand receptor interactions are involved in tumor immune infiltration and metastasis [37-39]. Therefore, these pathway analysis results may contribute to the comprehensive illumination of the hidden pathogenesis of IMPA.

FZD2 encodes a transmembrane protein with seven transmembrane domains and an extracellular cysteine-rich domain $[40,41]$. It can strongly bind to Wnt proteins, which subsequently activates the expression of downstream genes by the Wnt signaling pathway [40, 41]. A study performing a comprehensive analysis of FZD2 in 33 cancer types indicated that FZD2 was associated with high oncogenicity [42]. It was implicated to participate in cancer cell growth, migration and invasion and dominantly affected treatment and prognosis, suggesting the importance of FZD2 in 


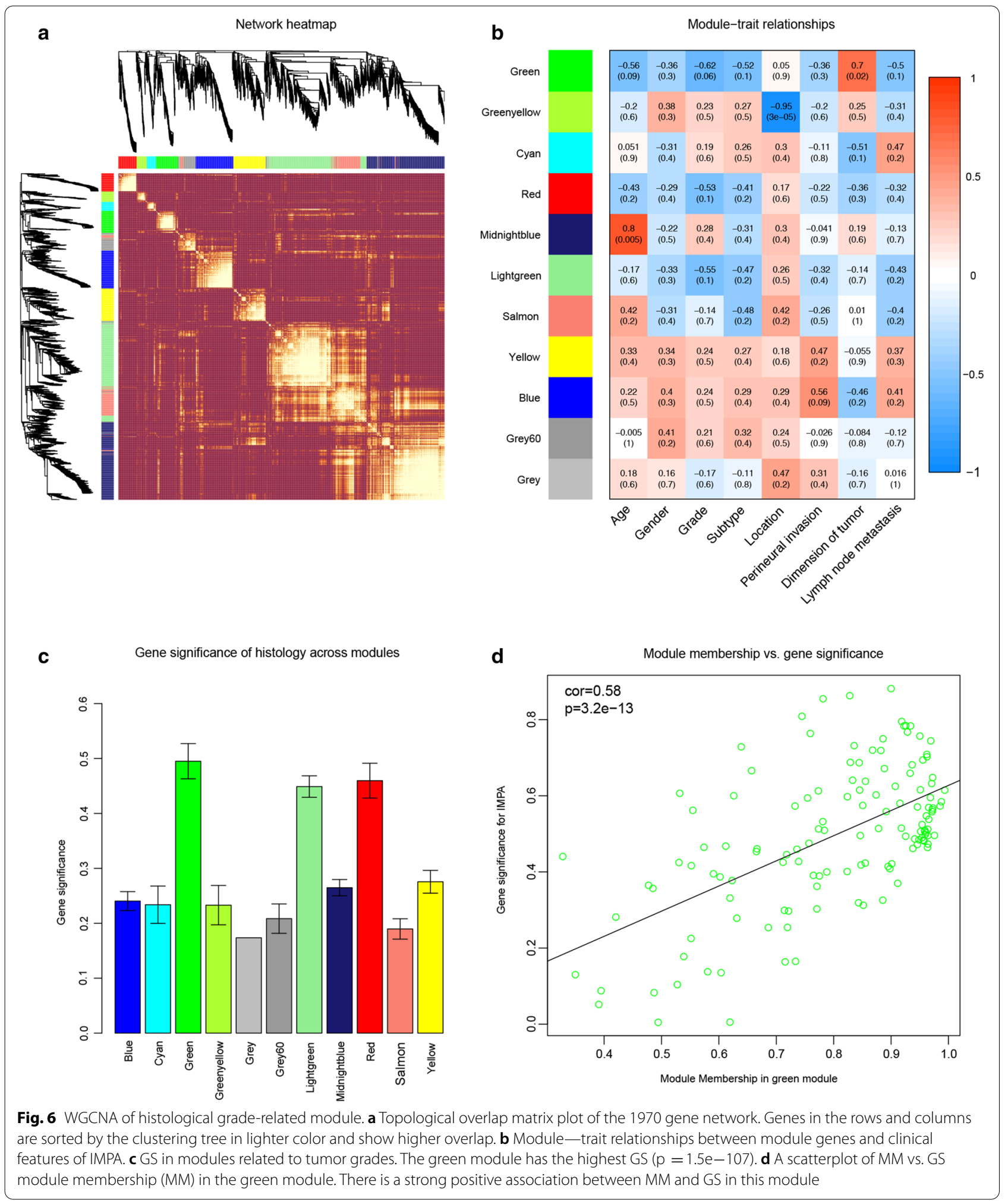




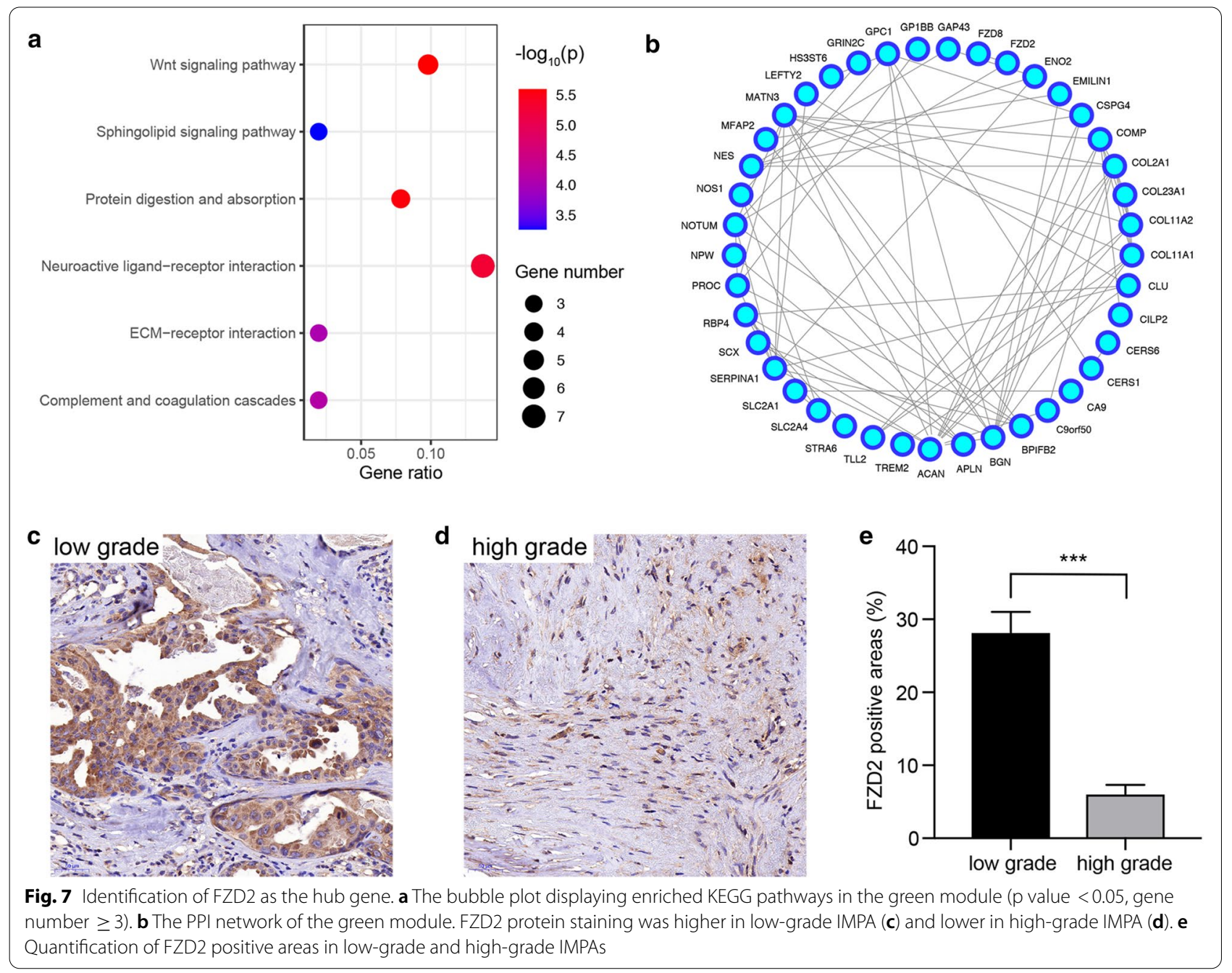

cancer [42]. In oral neoplasms, FZD2 promotes the oncogenesis of oral squamous cell carcinoma [43, 44]. Interestingly, FZD2 was found to act as an inhibitor in salivary adenoid cystic carcinoma, which might be due to the different microenvironment of these tumors [45]. Nonetheless, the role of FZD2 in malignant pleomorphic adenoma has not been reported till now.

In the present study, we showed that FZD2 in this module has the strongest relationship with the grade characteristics of IMPA. Some reports have demonstrated that the expression of FZD2 varies with the different clinical stages and histological grades in cancers $[42,46]$. Here, we first found that FZD2 was more highly upregulated in low-grade IMPA than in high-grade IMPA, indicating that FZD2 serves as an ideal indicator for the precise prediction of IMPA histological stages. In general, our study has uncovered an important role of FZD2 in the pathological progression of IMPA. Hub genes of the other 5 tumor-associated modules have also been screened out (Table 5), which might also play a role in IMPA. Further studies are necessary to figure out detailed functions of FZD2 as well as other 5 hub genes in IMPA.

\section{Conclusions}

The expression of FZD2 is negatively correlated with the clinical malignancy of IMPA. Moreover, FZD2 was identified as one of the hub genes in the IMPA transcription network and can play a vital role in regulating IMPA progression. In general, FZD2 serves as a promising indicator for predicting the histological stages of IMPA. 
Table 3 The intramodule connectivity values of 41 genes in the PPI network

\begin{tabular}{lclc}
\hline Gene_symbol & Intramodule & Gene_symbol & Intramodule \\
\hline ACAN & 52.61026603 & GP1BB & 32.09642238 \\
APLN & 49.50281239 & GPC1 & 17.64166846 \\
BGN & 12.90972979 & GRIN2C & 42.02411118 \\
BPIFB2 & 7.554629672 & HS3ST6 & 33.2011835 \\
C9orf50 & 54.8184171 & LEFTY2 & 20.31343892 \\
CA9 & 30.21814133 & MATN3 & 48.9472058 \\
CERS1 & 53.16205839 & MFAP2 & 34.5194173 \\
CERS6 & 10.1562834 & NES & 11.29861431 \\
CILP2 & 52.35240828 & NOS1 & 37.27240571 \\
CLU & 44.52886375 & NOTUM & 52.54787108 \\
COL11A1 & 17.74844867 & NPW & 51.46013624 \\
COL11A2 & 49.91357347 & PROC & 17.31603023 \\
COL23A1 & 16.6497107 & RBP4 & 54.28793703 \\
COL2A1 & 51.2326001 & SCX & 20.11143562 \\
COMP & 52.0206045 & SERPINA1 & 10.12214108 \\
CSPG4 & 15.87510143 & SLC2A1 & 6.839166971 \\
EMILIN1 & 51.4866436 & SLC2A4 & 33.68282253 \\
ENO2 & 33.90715954 & STRA6 & 23.69469976 \\
FZD2 & 56.74186498 & TLL2 & 19.18490841 \\
FZD8 & 24.15239668 & TREM2 & 10.95125665 \\
GAP43 & 15.30427057 & & \\
\hline & & & \\
& & & \\
& & & \\
& & &
\end{tabular}

Table 4 Association between FZD2 expression level and histological grade of IMPA

\begin{tabular}{lllll}
\hline Group & $\begin{array}{l}\text { High } \\
\text { expression of } \\
\text { FZD2 }\end{array}$ & $\begin{array}{l}\text { Low expression } \\
\text { of FZD2 }\end{array}$ & $\mathbf{X}^{\mathbf{2}}$ & p value \\
\hline High grade & 10 & 19 & 4.865 & $0.027^{*}$ \\
Low grade & 11 & 5 & & \\
\hline
\end{tabular}

Pearson's Chi-square test

*p value $<0.05$

Table 5 The hub genes of 6 tumor-associated gene modules

\begin{tabular}{|c|c|}
\hline Module & Hub gene \\
\hline Cyan ${ }^{\mathrm{a}}$ & IL6 \\
\hline Green & FZD2 \\
\hline Grey $60^{\mathrm{a}}$ & IL1B \\
\hline Midnight blue ${ }^{a}$ & AURKA \\
\hline Salmon ${ }^{a}$ & SRC \\
\hline Yellow ${ }^{\mathrm{a}}$ & CENPE \\
\hline
\end{tabular}

a Obtained by the Maximal Clique Centrality (MCC) algorithm of CytoHubba plugin

\section{Abbreviations}

IMPA: Invasive malignant pleomorphic adenoma; Ca-ex-PA: Carcinoma ex pleomorphic adenoma; RNA-seq: RNA sequencing; DWI: Diffusion-weighted imaging; $\mathrm{m}^{6} \mathrm{~A}$ : $\mathrm{N}^{6}$-methyladenosine; MeRIP-seq: Methylated RNA immunoprecipitation with high throughput sequencing; WGCNA: Weighted gene coexpression network analysis; PTC: Papillary thyroid carcinoma; H\&E: Hematoxylin and eosin; IHC: Immunohistochemistry; FZD2: Frizzled 2; DEGs: Differentially expressed genes; DAVID: Database for annotation, visualization and integrated discovery; PPI: Protein-protein interaction; GS: Gene significance; MM: Module membership; ME: Module eigengene; STRING: Retrieval of interacting genes/ proteins; BP: Biological process; ECM: Extracellular matrix.

\section{Supplementary Information}

The online version contains supplementary material available at https://doi. org/10.1186/s12967-021-03204-7.

Additional file 1: Table S1. The 1970 DEGs in IMPA and adjacent normal controls.

Additional file 2: Table S2. Table S2. GO and KEGG pathway analyses of 11 gene modules.

Additional file 3: Table S3. WGCNA results of the 1970 DEGs.

\section{Acknowledgements}

The authors would like to thank Professor Chunye Zhang and Dr. Biao Yang for their valuable assistance and instructions to improve this study.

\section{Authors' contributions}

ZT and QW conceived and developed the outline of the study and reviewed the draft of the paper. ZH, HR and JS performed the experiments, provided the statistical analysis and wrote the manuscript. $\amalg$ collected the IMPA samples and performed IHC. CG revised the manuscript. All authors read and approved the final manuscript.

\section{Funding}

The work was supported by the Natural Science Foundation of Shanghai (Grant No. 18ZR1422200).

\section{Availability of data and materials}

The datasets generated and/or analyzed during the current study are from the DAVID (https://david.ncifcrf.gov/). RNA-seq were uploaded to the GEO database (GSE161879 and GSE179895).

\section{Declarations}

\section{Ethics approval and consent to participate}

This study was approved by the Ethics Committee of Shanghai Ninth People's Hospital.

\section{Consent for publication \\ Not applicable.}

\section{Competing interests}

The authors declare that they have no competing interests.

\section{Author details}

${ }^{1}$ Department of Oral Pathology, Shanghai Ninth People's Hospital, Shanghai Jiao Tong University School of Medicine, Shanghai, China. ${ }^{2}$ National Clinical Research Center for Oral Diseases, Shanghai, China. ${ }^{3}$ Department of Oral and Maxillofacial Surgery, Peking University School and Hospital of Stomatology, Beijing, China. ${ }^{4}$ Department of Prosthodontics, School and Hospital of Stomatology, Cheeloo College of Medicine, Shandong University \& Shandong Provincial Key Laboratory of Oral Tissue Regeneration \& Shandong Engineering Laboratory for Dental Materials and Oral Tissue Regeneration, Jinan, Shandong, China. ${ }^{5}$ Pediatric Heart Center, Xinhua Hospital, School of Medicine, Shanghai Jiao Tong University, Shanghai, China. ${ }^{6}$ Clinical Translational Research 
Center, Shanghai Pulmonary Hospital, School of Life Sciences and Technology, Tongji University, Shanghai 200092, China.

Received: 3 August 2021 Accepted: 17 December 2021

Published online: 05 January 2022

\section{References}

1. Thompson L. World Health Organization classification of tumours: pathology and genetics of head and neck tumours. Ear Nose Throat J. 2006;85:74.

2. Hu Y, Xia L, Zhang C, Xia R, Tian Z, Li J. Clinicopathologic features and prognostic factors of widely invasive carcinoma ex pleomorphic adenoma of parotid gland: a clinicopathologic analysis of 126 cases in a Chinese population. J Oral Maxillofac Surg. 2020;78:2247-57.

3. Hashimoto K, Yamamoto H, Shiratsuchi H, Nakashima T, Tamiya S, Nishiyama K, Higaki Y, Komune S, Tsuneyoshi M, Oda Y. HER-2/neu gene amplification in carcinoma ex pleomorphic adenoma in relation to progression and prognosis: a chromogenic in-situ hybridization study. Histopathology. 2012;60:E131-142.

4. Kim JW, Kwon GY, Roh JL, Choi SH, Nam SY, Kim SY, Cho KJ. Carcinoma ex pleomorphic adenoma of the salivary glands: distinct clinicopathologic features and immunoprofiles between subgroups according to cellular differentiation. J Korean Med Sci. 2011;26:1277-85.

5. Di Palma S, Skálová A, VanìeekT, Simpson RH, Stárek I, Leivo I. Non-invasive (intracapsular) carcinoma ex pleomorphic adenoma: recognition of focal carcinoma by HER-2/neu and MIB1 immunohistochemistry. Histopathology. 2005;46:144-52.

6. Antony J, Gopalan V, Smith RA, Lam AK. Carcinoma ex pleomorphic adenoma: a comprehensive review of clinical, pathological and molecular data. Head Neck Pathol. 2012;6:1-9.

7. Shao S, Zheng N, Mao N, Xue X, Cui J, Gao P, Wang B. A triple-classification radiomics model for the differentiation of pleomorphic adenoma, Warthin tumour, and malignant salivary gland tumours on the basis of diffusionweighted imaging. Clin Radiol. 2021;76:472.e411-472.e418.

8. Hu YH, LiW, Zhang CY, Xia RH, Tian Z, Wang LZ, Xie L, Li J. Prognostic nomogram for disease-specific survival of carcinoma ex pleomorphic adenoma of the salivary gland. Head Neck. 2017;39:2416-24.

9. Han Z, Yang B, Wang Q, Hu Y, Wu Y, Tian Z. Comprehensive analysis of the transcriptome-wide $\mathrm{m}(6) \mathrm{A}$ methylome in invasive malignant pleomorphic adenoma. Cancer Cell Int. 2021;21:142.

10. Langfelder P, Horvath S. WGCNA: an R package for weighted correlation network analysis. BMC Bioinformatics. 2008;9:559.

11. Zhang B, Horvath S. A general framework for weighted gene co-expression network analysis. Stat Appl Genet Mol Biol. 2005;4:17.

12. Liu Y, Chen TY, Yang ZY, Fang W, Wu Q, Zhang C. Identification of hub genes in papillary thyroid carcinoma: robust rank aggregation and weighted gene co-expression network analysis. JTransl Med. 2020;18:170.

13. Tang J, Kong D, Cui Q, Wang K, Zhang D, Gong Y, Wu G. Prognostic genes of breast cancer identified by gene co-expression network analysis. Front Oncol. 2018:8:374.

14. Yang B, Dai JX, Pan YB, Ma YB, Chu SH. Examining the biomarkers and molecular mechanisms of medulloblastoma based on bioinformatics analysis. Oncol Lett. 2019;18:433-41.

15. Han Z, Zhuang X, Yang B, Jin L, Hong P, Xue J, Chen S, Tian Z. SYDE1 acts as an oncogene in glioma and has diagnostic and prognostic values. Front Mol Biosci. 2021:8:714203.

16. Robinson MD, McCarthy DJ, Smyth GK. edgeR: a bioconductor package for differential expression analysis of digital gene expression data. Bioinformatics. 2010;26:139-40.

17. da Huang W, Sherman BT, Lempicki RA. Systematic and integrative analysis of large gene lists using DAVID bioinformatics resources. Nat Protoc. 2009;4:44-57.

18. da Huang W, Sherman BT, Lempicki RA. Bioinformatics enrichment tools: paths toward the comprehensive functional analysis of large gene lists. Nucleic Acids Res. 2009;37:1-13.

19. The Gene Ontology resource. enriching a GOld mine. Nucleic Acids Res. 2021;49:D325-d334.
20. Kanehisa M, Furumichi M, Sato Y, Ishiguro-Watanabe M, Tanabe M. KEGG: integrating viruses and cellular organisms. Nucleic Acids Res. 2021;49:D545-d551.

21. Franceschini A, Szklarczyk D, Frankild S, Kuhn M, Simonovic M, Roth A, Lin J, Minguez P, Bork P, von Mering C, Jensen L. STRING V9.1: protein-protein interaction networks, with increased coverage and integration. Nucleic Acids Res. 2013;41:D808-815.

22. Szklarczyk D, Gable AL, Lyon D, Junge A, Wyder S, Huerta-Cepas J, Simonovic M, Doncheva NT, Morris JH, Bork P, et al. STRING v11: protein-protein association networks with increased coverage, supporting functional discovery in genome-wide experimental datasets. Nucleic Acids Res. 2019;47:D607-d613.

23. Shannon P, Markiel A, Ozier O, Baliga NS, Wang JT, Ramage D, Amin N, Schwikowski B, Ideker T. Cytoscape: a software environment for integrated models of biomolecular interaction networks. Genome Res. 2003;13:2498-504.

24. Yu G, Wang LG, Han Y, He QY. clusterProfiler: an R package for comparing biological themes among gene clusters. OMICS. 2012;16:284-7.

25. Wang Y, Jiang L, Dai G, Li S, Mu X. Bioinformatics analysis reveals different gene expression patterns in the annulus fibrosis and nucleus pulpous during intervertebral disc degeneration. Exp Ther Med. 2018;16:5031-40.

26. Piprek RP, Kloc M, Tassan JP, Kubiak JZ. Development of Xenopus laevis bipotential gonads into testis or ovary is driven by sex-specific cell-cell interactions, proliferation rate, cell migration and deposition of extracellular matrix. Dev Biol. 2017:432:298-310.

27. Vindin $\mathrm{H}$, Mithieux SM, Weiss AS. Elastin architecture. Matrix Biol. 2019:84:4-16.

28. Park CC, Zhang H, Pallavicini M, Gray JW, Baehner F, Park CJ, Bissell MJ. Beta1 integrin inhibitory antibody induces apoptosis of breast cancer cells, inhibits growth, and distinguishes malignant from normal phenotype in three dimensional cultures and in vivo. Cancer Res. 2006;66:1526-35.

29. Cao L, Xie B, Yang X, Liang H, Jiang X, Zhang D, Xue P, Chen D, Shao Z. MiR324-5p suppresses hepatocellular carcinoma cell invasion by counteracting ECM degradation through post-transcriptionally downregulating ETS1 and SP1. PLOS ONE. 2015;10:e0133074.

30. Kudla J, Becker D, Grill E, Hedrich R, Hippler M, Kummer U, Parniske M, Romeis T, Schumacher K. Advances and current challenges in calcium signaling. New Phytol. 2018;218:414-31.

31. Zheng X, Li X, Wang B, Cheng D, Li Y, Li W, Huang M, Tan X, Zhao G, Song $B$, et al. A systematic screen of conserved Ralstonia solanacearum effectors reveals the role of RipAB, a nuclear-localized effector that suppresses immune responses in potato. Mol Plant Pathol. 2019;20:547-61.

32. Sandal T, Aumo L, Hedin L, Gjertsen BT, Døskeland SO. Irod/lan5: an inhibitor of gamma-radiation- and okadaic acid-induced apoptosis. Mol Biol Cell. 2003;14:3292-304.

33. Bong AHL, Monteith GR. Calcium signaling and the therapeutic targeting of cancer cells. Biochim Biophys Acta Mol Cell Res. 2018;1865:1786-94.

34. Niehrs C. The complex world of WNT receptor signalling. Nat Rev Mol Cell Biol. 2012;13:767-79.

35. Zhan T, Rindtorff N, Boutros M. Wnt signaling in cancer. Oncogene. 2017;36:1461-73

36. Wang L, Sun T, Li S, Zhang Z, Jia J, Shan B. Protein anabolism is key to long-term survival in high-grade serous ovarian cancer. Transl Oncol. 2021;14:100885

37. Lu J, Hu F, Zhou Y. NR3C2-related transcriptome profile and clinical outcome in invasive breast carcinoma. Biomed Res Int. 2021;2021:9025481.

38. Yu J, Zhang Q, Wang M, Liang S, Huang H, Xie L, Cui C, Yu J. Comprehensive analysis of tumor mutation burden and immune microenvironment in gastric cancer. 2021. Biosci Rep. https://doi.org/10.1042/BSR20203336.

39. Qian X, Jiang C, Shen S, Zou X. GPRC5A: an emerging prognostic biomarker for predicting malignancy of pancreatic cancer based on bioinformatics analysis. J Cancer. 2021;12:2010-22.

40. Janda CY, Dang LT, You C, Chang J, de Lau W, Zhong ZA, Yan KS, Marecic O, Siepe D, Li X, et al. Surrogate Wnt agonists that phenocopy canonical Wnt and $\beta$-catenin signalling. Nature. 2017;545:234-7.

41. Fu Y, Zheng Q, Mao Y, Jiang X, Chen X, Liu P, Lv B, Huang T, Yang J, Cheng $Y$, et al. WNT2-mediated FZD2 stabilization regulates esophageal cancer metastasis via STAT3 signaling. Front Oncol. 2020;10:1168.

42. Zhou M, Sun X, Zhu Y. Analysis of the role of Frizzled 2 in different cancer types. FEBS Open Bio. 2021;11:1195-208. 
43. Zhang E, Li Z, Xu Z, Duan W, Sun C, Lu L. Frizzled 2 mediates the migration and invasion of human oral squamous cell carcinoma cells through the regulation of the signal transducer and activator of transcription-3 signaling pathway. Oncol Rep. 2015;34:3061-7.

44. Rhee CS, Sen M, Lu D, Wu C, Leoni L, Rubin J, Corr M, Carson DA. Wnt and frizzled receptors as potential targets for immunotherapy in head and neck squamous cell carcinomas. Oncogene. 2002;21:6598-605.

45. Ding LC, Huang XY, Zheng FF, Xie J, She L, Feng Y, Su BH, Zheng DL, Lu YG. FZD2 inhibits the cell growth and migration of salivary adenoid cystic carcinomas. Oncol Rep. 2016;35:1006-12.

46. Tuluhong D, Chen T, Wang J, Zeng H, Li H, Dunzhu W, Li Q, Wang S. FZD2 promotes TGF- $\beta$-induced epithelial-to-mesenchymal transition in breast cancer via activating notch signaling pathway. Cancer Cell Int. 2021;21:199.

\section{Publisher's Note}

Springer Nature remains neutral with regard to jurisdictional claims in published maps and institutional affiliations.

- fast, convenient online submission

- thorough peer review by experienced researchers in your field

- rapid publication on acceptance

- support for research data, including large and complex data types

- gold Open Access which fosters wider collaboration and increased citations

- maximum visibility for your research: over $100 \mathrm{M}$ website views per year

At BMC, research is always in progress.

Learn more biomedcentral.com/submissions 